\title{
An intrinsic nontriviality of graphs
}

\author{
RYO NIKKUNI
}

\begin{abstract}
We say that a graph is intrinsically nontrivial if every spatial embedding of the graph contains a nontrivial spatial subgraph. We prove that an intrinsically nontrivial graph is intrinsically linked, namely every spatial embedding of the graph contains a nonsplittable 2-component link. We also show that there exists a graph such that every spatial embedding of the graph contains either a nonsplittable 3-component link or an irreducible spatial handcuff graph whose constituent 2-component link is split.
\end{abstract}

57M15; 57M25

Dedicated to Professor Akio Kawauchi on his $60^{\text {th }}$ birthday

\section{Introduction}

Throughout this paper we work in the piecewise linear category. Let $f$ be an embedding of a finite graph $G$ into the 3 -sphere $\mathbb{S}^{3}$. Then $f$ (or $f(G)$ ) is called a spatial embedding of $G$ or simply a spatial graph. We call a subgraph $\gamma$ of $G$ which is homeomorphic to the circle a cycle. If $G$ is homeomorphic to the disjoint union of cycles, then $f$ is an $n$-component link (or knot if $n=1$ ). Two spatial embeddings $f$ and $g$ of $G$ are said to be ambient isotopic if there exists an orientation-preserving self homeomorphism $\Phi$ on $\mathbb{S}^{3}$ such that $\Phi \circ f=g$. A graph $G$ is said to be planar if there exists an embedding of $G$ into the 2-sphere, and a spatial embedding $f$ of a planar graph $G$ is said to be trivial if it is ambient isotopic to an embedding of $G$ into a 2 -sphere in $\mathbb{S}^{3}$. A spatial embedding $f$ of $G$ is said to be split if there exists a 2-sphere $S$ in $\mathbb{S}^{3}$ such that $S \cap f(G)=\varnothing$ and each connected component of $\mathbb{S}^{3} \backslash S$ has intersection with $f(G)$, and otherwise $f$ is said to be nonsplittable.

A graph $G$ is said to be intrinsically linked if every spatial embedding $f$ of $G$ contains a nonsplittable 2-component link. Conway and Gordon [2] and Sachs [15] showed that $K_{6}$ is intrinsically linked, where $K_{n}$ denotes the complete graph on $n$ vertices. Conway and Gordon also showed that $K_{7}$ is intrinsically knotted, namely every spatial embedding $f$ of $G$ contains a nontrivial knot. For a positive integer $n$, Flapan, Foisy, Naimi and Pommersheim [4] showed that there exists an intrinsically $n$-linked graph $G$, 
namely every spatial embedding $f$ of $G$ contains a nonsplittable $n$-component link (see also Flapan-Naimi-Pommersheim [3] and Bowlin-Foisy [1] for the case of $n=3$ ). Note that these results paid attention to only constituent knots and links of spatial graphs. Our purpose in this paper is to generalize the notion of intrinsically $n$-linkedness by paying attention to not only constituent knots and links but also spatial subgraphs which do not need to be knots and links, and to give graphs which have such a generalized property.

We say that a graph $G$ is intrinsically nontrivial if for every spatial embedding $f$ of $G$ there exists a planar subgraph $F$ of $G$ such that $\left.f\right|_{F}$ is not trivial. It is clear that intrinsically $n$-linked graphs are intrinsically nontrivial. Note that $F$ depends on $f$ and does not need to have the same topological type uniformly. In this situation, pioneering work has been done by Foisy [5]. Let $P$ and $P^{\prime}$ be graphs in the Petersen family, which is a family of seven graphs obtained from $K_{6}$ by $\nabla-Y$ or $Y-\nabla$ exchanges (see Sachs [15]); all intrinsically linked graphs have a minor in this family by Robertson-Seymour-Thomas [14]. Let $P *_{4} P^{\prime}$ be the graph which consists of $P$ and $P^{\prime}$ connected by four disjoint edges $e_{1}, e_{2}, e_{3}$ and $e_{4}$ as illustrated in Figure 1, which shows the case of $P=P^{\prime}=K_{6}$. Then he showed that $P *_{4} P^{\prime}$ is intrinsically knotted or 3-linked, namely every spatial embedding of $P *_{4} P^{\prime}$ contains either a nontrivial knot or a nonsplittable 3 -component link. He also showed that $K_{6} *_{4} K_{6}$ is neither intrinsically knotted nor intrinsically 3 -linked.

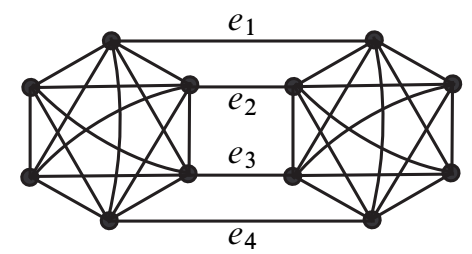

Figure 1: $K_{6} *_{4} K_{6}$

It was already known that intrinsically knotted graphs and intrinsically $n$-linked graphs $(n \geq 3)$ are intrinsically linked by Robertson-Seymour-Thomas' characterization of intrinsically linked graphs [14]. First, we show that if a graph $G$ is intrinsically nontrivial then every spatial embedding of $G$ must contain a nonsplittable 2-component link as follows.

Theorem 1.1 Intrinsically nontrivial graphs are intrinsically linked.

Then, for an intrinsically nontrivial graph $G$, we are interested in the collection of nontrivial spatial graph types (except for 2-component link types) where every spatial 
embedding of $G$ contains at least one spatial graph type in the collection. Foisy's above result also only paid attention to constituent knots and links of spatial graphs. We shall consider a smaller graph than $P *_{4} P^{\prime}$ from the viewpoint of intrinsic nontriviality. Let $P *_{3} P^{\prime}$ be the graph which is obtained from $P *_{4} P^{\prime}$ by deleting $e_{4}$. Then we have the following.

Theorem 1.2 Let $P$ and $P^{\prime}$ be graphs in the Petersen family. Then every spatial embedding of $P *_{3} P^{\prime}$ contains either a nonsplittable 3-component link or an irreducible spatial handcuff graph whose constituent 2-component link is split.

Here a spatial handcuff graph is a spatial embedding $f$ of the graph $H$ which is illustrated in Figure 2. Note that an orientation is given to each loop, namely we regard $f\left(\gamma_{1} \cup \gamma_{2}\right)$ as an ordered and oriented 2-component link. A spatial handcuff graph $f$ is said to be irreducible if there does not exist a 2 -sphere in $\mathbb{S}^{3}$ which intersects $f(H)$ transversely at one point. Note that an irreducible spatial handcuff graph is not trivial. We also show that $K_{6} *_{3} K_{6}$ has a spatial embedding which does not contain a nonsplittable 3-component link, and another spatial embedding which does not contain an irreducible spatial handcuff graph whose constituent 2-component link is split (Example 4.4). In particular, the former spatial embedding does not contain a nontrivial knot or a nonsplittable $n$-component link for $n \geq 3$. Namely Theorem 1.2 gives a new type of intrinsic nontriviality of graphs which cannot be detected by observing only constituent knots and links of its spatial embeddings.

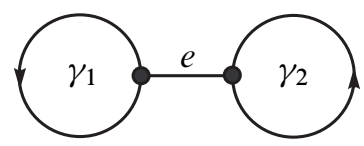

Figure 2

In the next section, we prove Theorem 1.1. In Section 3, we recall an ambient isotopy invariant of spatial handcuff graphs which was introduced by the author in [11]. In Section 4, we prove Theorem 1.2.

\section{Proof of Theorem 1.1}

A spatial embedding $f$ of a graph $G$ is said to be free if the fundamental group of the spatial graph complement $\pi_{1}\left(\mathbb{S}^{3} \backslash f(G)\right)$ is a free group. 
We say that $G$ is intrinsically nonfree if for every spatial embedding of $G$ there exists a subgraph $F$ of $G$ such that $\left.f\right|_{F}$ is not free. To prove Theorem 1.1, we show the following.

Theorem 2.1 The following are equivalent.

(1) $G$ is intrinsically nonfree.

(2) $G$ is intrinsically nontrivial.

(3) $G$ is intrinsically linked.

Proof It is clear that (3) implies (2). Next we show that (2) implies (1). If $G$ is intrinsically nontrivial, we have that for every spatial embedding $f$ of $G$ there exists a planar subgraph $F$ of $G$ such that $\left.f\right|_{F}$ is nontrivial. Then by ScharlemannThompson's famous criterion [16], there exists a subgraph $F^{\prime}$ of $F$ such that $\left.f\right|_{F^{\prime}}$ is not free. Since $F^{\prime}$ is also a subgraph of $G$, we have that $G$ is intrinsically nonfree. Finally we show that (1) implies (3). Assume that $G$ is not intrinsically linked. Then it follows from Robertson-Seymour-Thomas [14, (1.2)] that there exists a spatial embedding $f$ of $G$ such that for any cycle $\gamma$ of $G$ there exists a 2-disk $D_{\gamma}$ in $\mathbb{S}^{3}$ such that $f(G) \cap D_{\gamma}=f(G) \cap \partial D_{\gamma}=f(\gamma)$. At this time, it is also known that $\left.f\right|_{F}$ is free for any subgraph $F$ of $G$ [14, (3.3)] (the case that $G$ is planar was first shown by $\mathrm{Wu}[20])$. Thus we have that $G$ is not intrinsically nonfree.

\section{An invariant of spatial handcuff graphs}

In this section we give the definition of an invariant of spatial handcuff graphs which can detect an irreducible one whose constituent 2-component link is split. Let $L=J_{1} \cup J_{2}$ be an ordered and oriented 2-component link. Let $D$ be an oriented 2-disk and $x_{1}$, $x_{2}$ disjoint arcs in $\partial D$, where $\partial D$ has the orientation induced by the one of $D$, and each arc has an orientation induced by the one of $\partial D$. We assume that $D$ is embedded in $\mathbb{S}^{3}$ so that $D \cap L=x_{1} \cup x_{2}$ and $x_{i} \subset J_{i}$ with opposite orientations for each $i$. Then we call a knot $K_{D}=(L \cup \partial D) \backslash$ (int $x_{1} \cup$ int $\left.x_{2}\right)$ a $D-$ sum of $L$. For a spatial handcuff graph $f$, we denote $f\left(\gamma_{1} \cup \gamma_{2}\right)$ by $L_{f}$ and consider a $D$-sum of $L_{f}$ so that $f(e) \subset D, f(e) \cap \partial D=f(e) \cap L_{f}=\left\{p_{1}, p_{2}\right\}$ and $p_{i} \in$ int $x_{i}(i=1,2)$. We call such a $D$-sum of $L_{f}$ a $D$-sum of $L_{f}$ with respect to $f$ and denote it by $K_{D}(f)$. Though $K_{D}(f)$ is not uniquely determined up to ambient isotopy, the author showed in [11, Remark $3.4(1)]$ that the modulo $\operatorname{lk}\left(L_{f}\right)$ reduction of $a_{2}\left(K_{D}(f)\right)$ is an ambient isotopy invariant of $f$, where $\mathrm{lk}$ denotes the linking number in $\mathbb{S}^{3}$ and $a_{2}$ denotes the second coefficient of the Conway polynomial. Then we define

$$
n(f, D) ;=a_{2}\left(K_{D}(f)\right)-a_{2}\left(f\left(\gamma_{1}\right)\right)-a_{2}\left(f\left(\gamma_{2}\right)\right)
$$


and denote the modulo $\operatorname{lk}\left(L_{f}\right)$ reduction of $n(f, D)$ by $\bar{n}(f)$. It is clear that $\bar{n}(f)$ is also an ambient isotopy invariant of $f$. In particular, $\bar{n}(f)$ is a uniquely determined integer if $\operatorname{lk}\left(L_{f}\right)=0$. In this case we denote $\bar{n}(f)$ by $n(f)$ simply. Then we have the following.

Lemma 3.1 Let $f$ be a spatial handcuff graph. If $f$ is not irreducible, then for every choice of $D, n(f, D)=0$. In particular, if $\operatorname{lk}\left(L_{f}\right)=0$, then $f$ is irreducible if $n(f) \neq 0$.

Proof If $f$ is not irreducible, then $L_{f}$ is split and any $D$-sum of $L_{f}$ with respect to $f$ is the connected sum of $f\left(\gamma_{1}\right)$ and $f\left(\gamma_{2}\right)$. Recall that $a_{2}$ is additive under the connected sum of knots [7]. Thus we have that

$$
\begin{aligned}
n(f, D) & =a_{2}\left(K_{D}(f)\right)-a_{2}\left(f\left(\gamma_{1}\right)\right)-a_{2}\left(f\left(\gamma_{2}\right)\right) \\
& =a_{2}\left(f\left(\gamma_{1}\right) \sharp f\left(\gamma_{2}\right)\right)-a_{2}\left(f\left(\gamma_{1}\right)\right)-a_{2}\left(f\left(\gamma_{2}\right)\right) \\
& =a_{2}\left(f\left(\gamma_{1}\right)\right)+a_{2}\left(f\left(\gamma_{2}\right)\right)-a_{2}\left(f\left(\gamma_{1}\right)\right)-a_{2}\left(f\left(\gamma_{2}\right)\right) \\
& =0 .
\end{aligned}
$$

Therefore we have the result.

For integers $r$ and $s$, let $f_{r, s}$ be the spatial handcuff graph as illustrated in Figure 3, where the rectangles represented by $r$ and $s$ stand for $|r|$ full twists and $|s|$ full twists

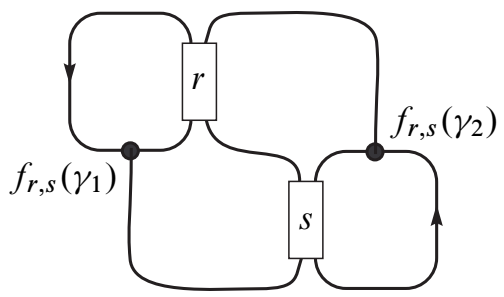

Figure 3

as illustrated in Figure 4, respectively. Note that the constituent 2-component link $L_{f_{r, s}}$ is trivial. Then we have the following.

Lemma 3.2 $n\left(f_{r, s}\right)=2 r s$.

Proof Let $K_{+}, K_{-}$and $K_{0}$ be two oriented knots and an oriented 2-component link which are identical except inside the depicted regions as illustrated in Figure 5. 


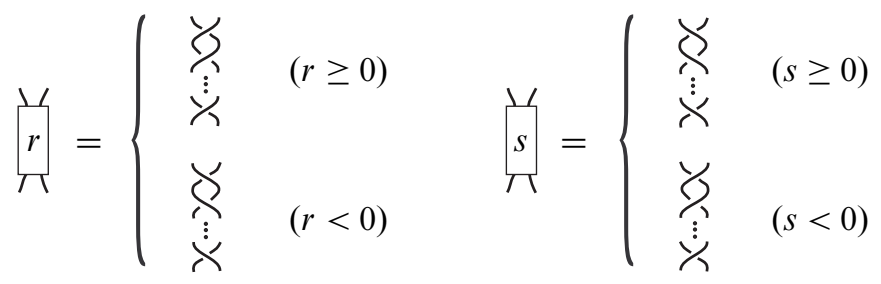

Figure 4

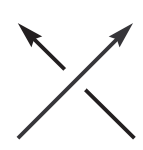

$K_{+}$

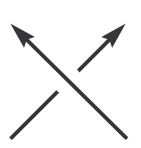

$K_{-}$

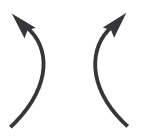

$K_{0}$

Figure 5

Then it is well known that $a_{2}\left(K_{+}\right)-a_{2}\left(K_{-}\right)=1 \mathrm{k}\left(K_{0}\right)$ [6]. We consider the $D$-sum of $L_{f}$ with respect to $f_{r, s}$ and the skein tree started from $K_{D}\left(f_{r, s}\right)$ as illustrated in Figure 6 , where $\varepsilon= \pm 1$ is the usual sign of marked crossing. Note that $\varepsilon=1$ if $r>0$ and -1 if $r<0$. Then

$$
\begin{aligned}
a_{2}\left(K_{D}\left(f_{r, s}\right)\right)-a_{2}(J) & =\varepsilon \operatorname{lk}\left(M_{1}\right)=\varepsilon(r-\varepsilon+s), \\
a_{2}\left(K_{D}\left(f_{r-\varepsilon, s}\right)\right)-a_{2}(J) & =\varepsilon \operatorname{lk}\left(M_{2}\right)=\varepsilon(r-\varepsilon-s) .
\end{aligned}
$$

Thus by (3-1) and (3-2),

$$
a_{2}\left(K_{D}\left(f_{r, s}\right)\right)-a_{2}\left(K_{D}\left(f_{r-\varepsilon, s}\right)\right)=2 \varepsilon s .
$$

Hence we have that

$$
\begin{aligned}
a_{2}\left(K_{D}\left(f_{r, s}\right)\right) & =a_{2}\left(K_{D}\left(f_{r-\varepsilon, s}\right)\right)+2 \varepsilon s \\
& =a_{2}\left(K_{D}\left(f_{r-2 \varepsilon, s}\right)\right)+2 \varepsilon s+2 \varepsilon s \\
& \vdots \\
& =a_{2}\left(K_{D}\left(f_{r-|r| \varepsilon, s}\right)\right)+2 \varepsilon|r| s \\
& =a_{2}\left(K_{D}\left(f_{0, s}\right)\right)+2 r s .
\end{aligned}
$$




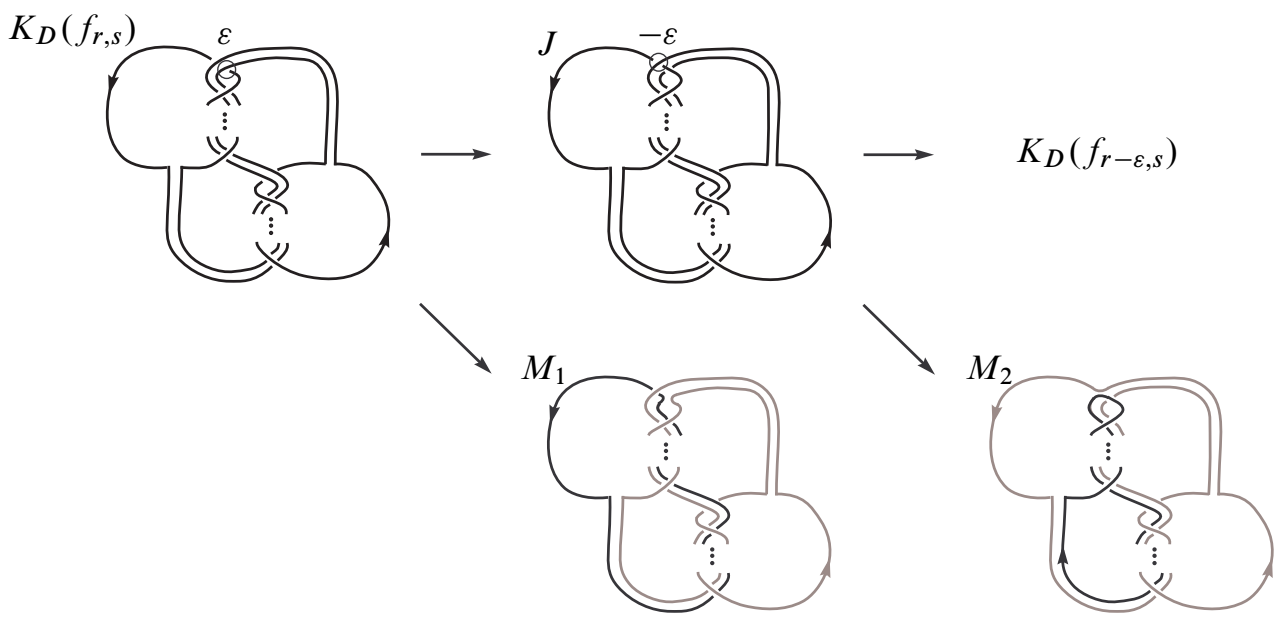

Figure 6

It is easy to see that $f_{0, s}$ is trivial, namely $a_{2}\left(K_{D}\left(f_{0, s}\right)\right)=0$. Therefore by (3-3),

$$
n\left(f_{r, s}\right)=a_{2}\left(K_{D}\left(f_{r, s}\right)\right)-a_{2}\left(f_{r, s}\left(\gamma_{1}\right)\right)-a_{2}\left(f_{r, s}\left(\gamma_{2}\right)\right)=2 r s .
$$

This completes the proof.

By Lemma 3.1 and Lemma 3.2, we have that if $r, s \neq 0$ then $f_{r, s}$ is irreducible. Since $L_{f_{r, s}}$ is trivial, $\left\{f_{r, s}\right\}_{r, s \neq 0}$ is a family of minimally knotted spatial handcuff graphs, namely each $f_{r, s}$ is nontrivial and any of whose spatial proper subgraphs is trivial.

\section{Proof of Theorem 1.2}

Let $P_{4}$ be the oriented graph consists of four edges $e_{1}, e_{2} e_{3}, e_{4}$ and two loops $e_{5}, e_{6}$ as illustrated in Figure 7. We denote the cycles $e_{5}, e_{1} \cup e_{2}, e_{3} \cup e_{4}$ and $e_{6}$ of $P_{4}$ by $c_{1}, c_{2}, c_{3}$ and $c_{4}$, respectively, and the subgraph $c_{1} \cup e_{i} \cup e_{j} \cup c_{4}(i=1,2, j=3,4)$ by $H_{i j}$. Note that $H_{i j}$ is homeomorphic to the graph $H$ illustrated in Figure 2. Let $f$ be a spatial embedding of $P_{4}$ with $\operatorname{lk}\left(f\left(c_{1} \cup c_{4}\right)\right)=0$. Then we define $\xi(f) \in \mathbb{Z}$ by

$$
\begin{aligned}
\xi(f) & =\sum_{i, j}(-1)^{i+j} n\left(\left.f\right|_{H_{i j}}\right) \\
& =n\left(\left.f\right|_{H_{13}}\right)-n\left(\left.f\right|_{H_{14}}\right)-n\left(\left.f\right|_{H_{23}}\right)+n\left(\left.f\right|_{H_{24}}\right) .
\end{aligned}
$$

Proposition 4.1 $\xi(f)$ is a Delta equivalence invariant of $f$. 


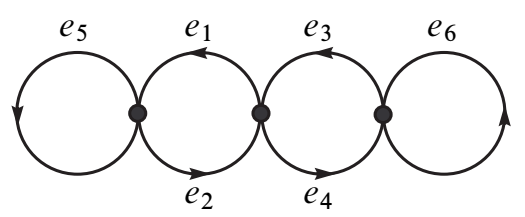

Figure 7

Here, a Delta equivalence is an equivalence relation on spatial graphs which is generated by Delta moves and ambient isotopies, where a Delta move is a local move on a spatial graph as illustrated in Figure 8 [8; 10]. It is shown in [9] that a Delta equivalence

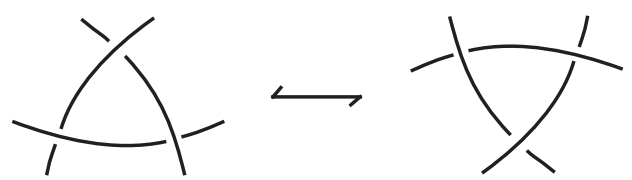

Figure 8

coincides with a (spatial graph-)homology, which is an equivalence relation on spatial graphs introduced in [18]. Note that a Delta move preserves the linking number of each of the constituent 2-component links. We show the following lemma needed to prove Proposition 4.1.

Lemma 4.2 Let $g$ be a spatial handcuff graph with $\operatorname{lk}\left(L_{g}\right)=0$ and $h$ a spatial handcuff graph obtained from $g$ by a single Delta move. Then we have the following.

(1) If either $g\left(\gamma_{1}\right)$ or $g\left(\gamma_{2}\right)$ does not appear in the Delta move as the strings, then $n(g)=n(h)$.

(2) If both $g\left(\gamma_{1}\right)$ and $g\left(\gamma_{2}\right)$ appear and $g(e)$ does not appear in the Delta move as the strings, then $n(g)-n(h)= \pm 1$.

(3) If all of $g\left(\gamma_{1}\right), g\left(\gamma_{2}\right)$ and $g(e)$ appear in the Delta move as the strings, then $n(g)-n(h)= \pm 2$ or 0 .

Proof (1) If all of the three strings in such an intended Delta move belong to the same spatial edge (such a move is called a self Delta move), we have the result because it is known that $n(g)$ is invariant under a self Delta move [11, Theorem 2.1]. Next we consider the case that at least one of the three strings in the Delta move belong to $g(e)$. If $g\left(\gamma_{j}\right)$ does not appear in the Delta move as the strings $(j=1$ or 2$)$, then by 
applying the deformation on $g(H)$ as illustrated in Figure 9 repeatedly, we can see that such a Delta move may be realized by self Delta moves on $g\left(\gamma_{i}\right)(i \neq j)$. Therefore we have that $n(g)=n(h)$.

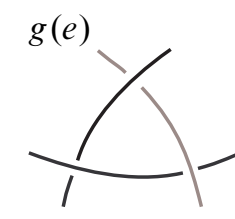

$\underset{\text { isotopic }}{\text { ambient }}$

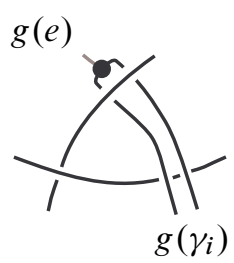

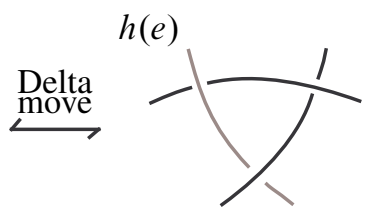

$\eta \underset{\text { isotopic }}{\text { ambient }}$

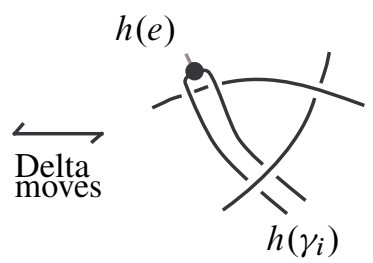

Figure 9

(2) If $h$ is obtained from $g$ by such an intended single Delta move, then we may consider $K_{D}(g)$ and $K_{D}(h)$ by the same 2-disk $D$ so that $K_{D}(h)$ is obtained from $K_{D}(g)$ by a single Delta move. Then by the result of Okada [13] that if a knot $K_{1}$ is obtained from a knot $K_{2}$ by a single delta move then $a_{2}\left(K_{1}\right)-a_{2}\left(K_{2}\right)= \pm 1$, we have that $a_{2}\left(K_{D}(g)\right)-a_{2}\left(K_{D}(h)\right)= \pm 1$. On the other hand, since a Delta move is a 3-component Brunnian local move [19, Section 2, Examples (2)], we have that $g\left(\gamma_{i}\right)$ and $h\left(\gamma_{i}\right)$ are ambient isotopic $(i=1,2)$. Hence we have that $n(g)-n(h)=a_{2}\left(K_{D}(g)\right)-a_{2}\left(K_{D}(h)\right)= \pm 1$.

(3) If $h$ is obtained from $g$ by such an intended single Delta move, then we may consider $K_{D}(g)$ and $K_{D^{\prime}}(h)$ so that $K_{D^{\prime}}(h)$ is obtained from $K_{D}(g)$ by twice Delta moves as illustrated in Figure 10. Thus by Okada's result as we said in the proof of (2), we have that $a_{2}\left(K_{D}(g)\right)-a_{2}\left(K_{D^{\prime}}(h)\right)= \pm 2$ or 0 . Note that $g\left(\gamma_{i}\right)$ and $h\left(\gamma_{i}\right)$ are ambient isotopic $(i=1,2)$ by Brunnian property of the Delta move. Hence we have that $n(g)-n(h)=a_{2}\left(K_{D}(g)\right)-a_{2}\left(K_{D^{\prime}}(h)\right)= \pm 2$ or 0 .

Proof of Proposition 4.1 Let $f^{\prime}$ be a spatial handcuff graph which is obtained from $f$ by a single Delta move. It is sufficient to show that $\xi(f)=\xi\left(f^{\prime}\right)$. If either $f\left(e_{5}\right)$ or $f\left(e_{6}\right)$ does not appear in the Delta move as the strings, then by Lemma 4.2 (1) 


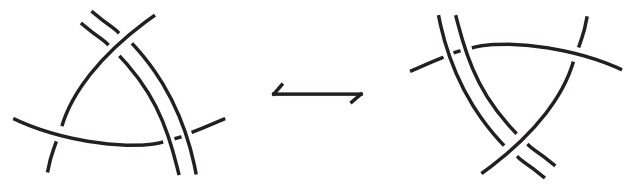

Figure 10

and Brunnian property of the Delta move, we have that $n\left(\left.f\right|_{H_{i j}}\right)=n\left(\left.f^{\prime}\right|_{H_{i j}}\right)$ for any $i=1,2$ and $j=3,4$. Hence $\xi(f)=\xi\left(f^{\prime}\right)$. If none of $f\left(e_{1}\right), f\left(e_{2}\right), f\left(e_{3}\right)$ or $f\left(e_{4}\right)$ appears in the Delta move as the strings, then by Lemma $4.2(2)$ we have that $n\left(\left.f\right|_{H_{i j}}\right)-n\left(\left.f^{\prime}\right|_{H_{i j}}\right)= \pm 1(i=1,2, j=3,4)$. Note that if two oriented knots differ by a single Delta move then the variation of $a_{2}$ of them is determined only by the order of strings in the Delta move and their orientations arising from following the knot along the orientation (cf [12;21, Theorem 6]). Hence we have that $n\left(\left.f\right|_{H_{i j}}\right)-n\left(\left.f^{\prime}\right|_{H_{i j}}\right)=1$ $(i=1,2, j=3,4)$ or $n\left(\left.f\right|_{H_{i j}}\right)-n\left(\left.f^{\prime}\right|_{H_{i j}}\right)=-1(i=1,2, j=3,4)$. Then

$$
\xi(f)-\xi\left(f^{\prime}\right)=\sum_{i, j}(-1)^{i+j}\left(n\left(\left.f\right|_{H_{i j}}\right)-n\left(\left.f^{\prime}\right|_{H_{i j}}\right)\right)=( \pm 1) \sum_{i, j}(-1)^{i+j}=0 .
$$

Finally we consider the case that all of $f\left(e_{5}\right), f\left(e_{6}\right)$ and $f\left(e_{k}\right)$ appear in the Delta move as the strings $(k=1,2,3,4)$. It is sufficient to check the case of $k=1$. Then by Lemma 4.2 (3) and noting as before the variation of $a_{2}$ of two oriented knots differing by a single Delta move, it holds that any one of $n\left(\left.f\right|_{H_{1 j}}\right)-n\left(\left.f^{\prime}\right|_{H_{1 j}}\right)=2(j=3,4)$, $n\left(\left.f\right|_{H_{1 j}}\right)-n\left(\left.f^{\prime}\right|_{H_{1 j}}\right)=-2(j=3,4)$ or $n\left(\left.f\right|_{H_{1 j}}\right)-n\left(\left.f^{\prime}\right|_{H_{1 j}}\right)=0(j=3,4)$. Note that $\left.f\right|_{H_{2 j}}$ and $\left.f^{\prime}\right|_{H_{2 j}}$ are ambient isotopic for any $j=3,4$ by Brunnian property of the Delta move. Then

$$
\xi(f)-\xi\left(f^{\prime}\right)=n\left(\left.f\right|_{H_{13}}\right)-n\left(\left.f^{\prime}\right|_{H_{13}}\right)-\left(n\left(\left.f\right|_{H_{14}}\right)-n\left(\left.f^{\prime}\right|_{H_{14}}\right)\right)=0 .
$$

Therefore we have the desired conclusion.

Lemma 4.3 Let $f$ be a spatial embedding of $P_{4}$ with $\operatorname{lk}\left(f\left(c_{1} \cup c_{4}\right)\right)=0$. Then we have that $\operatorname{lk}\left(f\left(c_{1} \cup c_{3}\right)\right) \operatorname{lk}\left(f\left(c_{2} \cup c_{4}\right)\right) \neq 0$ if and only if $\xi(f) \neq 0$.

Proof It is known that two spatial embeddings of a planar graph are Delta equivalent if and only if their corresponding constituent 2-component links have the same linking number [17]. Therefore we have that if $\operatorname{lk}\left(f\left(c_{1} \cup c_{3}\right)\right)=s$ and $\operatorname{lk}\left(f\left(c_{2} \cup c_{4}\right)\right)=r$, then $f$ is Delta equivalent to the spatial embedding $h_{r, s}$ of $P_{4}$ as illustrated in Figure 11, where the rectangles represented by $r$ and $s$ stand for $|r|$ full twists and $|s|$ full twists as illustrated in Figure 4, respectively. Note that $\left.h_{r, s}\right|_{H_{13}},\left.h_{r, s}\right|_{H_{14}}$ and $\left.h_{r, s}\right|_{H_{23}}$ are 


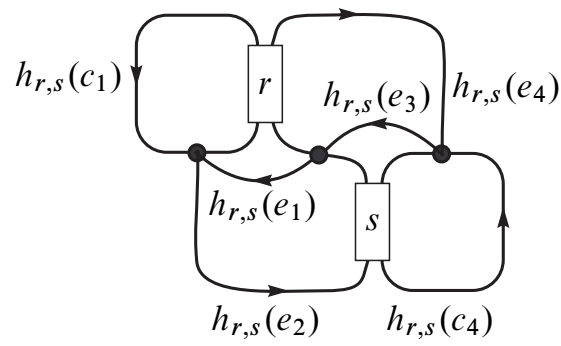

Figure 11

trivial spatial handcuff graphs. Then by combining Proposition 4.1 and Lemma 3.2,

$$
\xi(f)=\xi\left(h_{r, s}\right)=n\left(\left.h_{r, s}\right|_{H_{24}}\right)=2 r s .
$$

This implies the result.

Note that if $\xi(f) \neq 0$ then there must exist a subgraph $H_{i j}$ such that $n\left(\left.f\right|_{H_{i j}}\right) \neq 0$. Then by Lemma 3.1 we have that $\left.f\right|_{H_{i j}}$ is irreducible.

Proof of Theorem 1.2 Let $f$ be a spatial embedding of $P *_{3} P^{\prime}$. Note that every spatial embedding of a graph in the Petersen family contains a 2-component link with odd linking number $[2 ; 19]$. Hence there exists a pair of two disjoint cycles $\gamma_{1}, \gamma_{2}$ of $P$ such that $\operatorname{lk}\left(f\left(\gamma_{1} \cup \gamma_{2}\right)\right) \neq 0$, and there exists a pair of two disjoint cycles $\gamma_{1}^{\prime}, \gamma_{2}^{\prime}$ of $P^{\prime}$ such that $\operatorname{lk}\left(f\left(\gamma_{1}^{\prime} \cup \gamma_{2}^{\prime}\right)\right) \neq 0$. If there exist two edges $e_{i}$ and $e_{j}$ $(1 \leq i<j \leq 3)$ each of which connects $\gamma_{l}$ with $\gamma_{k}^{\prime}$ for some $l$ and $k$, by BowlinFoisy's argument [1, Lemma 3], we have that $f\left(\gamma_{1} \cup \gamma_{2} \cup \gamma_{1}^{\prime} \cup \gamma_{2}^{\prime} \cup e_{i} \cup e_{j}\right)$ contains a nonsplittable 3-component link. If for any $l$ and $k$ there do not exist two edges $e_{i}$ and $e_{j}(1 \leq i<j \leq 3)$ each of which connects $\gamma_{l}$ with $\gamma_{k}^{\prime}$, then we may assume that $e_{1}$ connects $\gamma_{1}$ with $\gamma_{1}^{\prime}, e_{2}$ connects $\gamma_{1}$ with $\gamma_{2}^{\prime}$ and $e_{3}$ connects $\gamma_{2}$ with $\gamma_{2}^{\prime}$ without loss of generality. If $f\left(\gamma_{2} \cup \gamma_{1}^{\prime}\right)$ is nonsplittable, then $f\left(\gamma_{1} \cup \gamma_{2} \cup \gamma_{1}^{\prime}\right)$ is a nonsplittable 3-component link. If $f\left(\gamma_{2} \cup \gamma_{1}^{\prime}\right)$ is split, then let us consider the subgraph $F=\gamma_{1} \cup \gamma_{2} \cup \gamma_{1}^{\prime} \cup \gamma_{2}^{\prime} \cup e_{1} \cup e_{2} \cup e_{3}$ of $P *_{3} P^{\prime}$. We denote the graph obtained from $F$ by contracting $e_{1}, e_{2}$ and $e_{3}$ by $F / e_{1} / e_{2} / e_{3}$. Note that $F / e_{1} / e_{2} / e_{3}$ is homeomorphic to $P_{4}$ which is illustrated in Figure 7. Let $\bar{f}$ is a spatial embedding of $F / e_{1} / e_{2} / e_{3}$ naturally induced from $\left.f\right|_{F}$. Then by applying Lemma 4.3 and Lemma 3.1 to $\bar{f}$, we have that $\bar{f}\left(F / e_{1} / e_{2} / e_{3}\right)$ contains an irreducible spatial handcuff graph whose constituent 2-component link is split. Then it is clear that $f(F)$ also contains a spatial handcuff graph with the same spatial graph type as above. This implies that $f$ contains an irreducible spatial handcuff graph whose constituent 2-component link is split. This completes the proof. 
Example 4.4 Let $f$ be the spatial embedding of $K_{6} *_{3} K_{6}$ as illustrated in Figure 12 . Then we can see that each of the spatial handcuff graphs contained in $f$ is trivial or ambient isotopic to the spatial handcuff graph as illustrated in Figure 13. Namely $f$ does not contain an irreducible spatial handcuff graph whose constituent 2-component link is split. But $f$ contains exactly one nonsplittable 3-component link. Next, let $g$ be the spatial embedding of $K_{6} *_{3} K_{6}$ as illustrated in Figure 12 which is obtained from $f$ by a single crossing change. Then we can see that each of the constituent knots of $g$ is trivial and each of the constituent $n$-component links of $g$ is split for $n \geq 3$. But $g$ contains exactly one irreducible spatial handcuff graph whose constituent 2-component link is split.
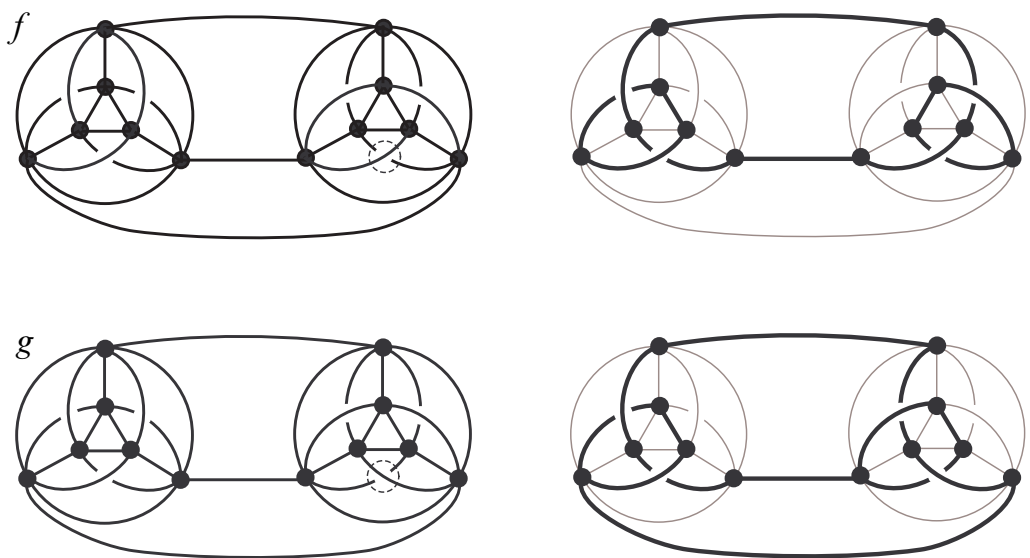

Figure 12

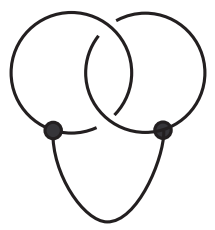

Figure 13

Acknowledgments The author was partially supported by Grant-in-Aid for Young Scientists (B) (No. 18740030), Japan Society for the Promotion of Science. He is also grateful to the referee for his or her comments. 


\section{References}

[1] G Bowlin, J Foisy, Some new intrinsically 3-linked graphs, J. Knot Theory Ramifications 13 (2004) 1021-1027 MR2108646

[2] J H Conway, CM Gordon, Knots and links in spatial graphs, J. Graph Theory 7 (1983) 445-453 MR722061

[3] E Flapan, R Naimi, J Pommersheim, Intrinsically triple linked complete graphs, Topology Appl. 115 (2001) 239-246 MR1847466

[4] E Flapan, J Pommersheim, J Foisy, R Naimi, Intrinsically n-linked graphs, J. Knot Theory Ramifications 10 (2001) 1143-1154 MR1871222

[5] J Foisy, Graphs with a knot or 3-component link in every spatial embedding, J. Knot Theory Ramifications 15 (2006) 1113-1118 MR2287434

[6] L H Kauffman, Formal knot theory, Math. Notes 30, Princeton Univ. Press (1983) MR712133

[7] L H Kauffman, On knots, Annals of Math. Studies 115, Princeton Univ. Press (1987) MR907872

[8] S V Matveev, Generalized surgeries of three-dimensional manifolds and representations of homology spheres, Mat. Zametki 42 (1987) 268-278, 345 MR915115

[9] T Motohashi, K Taniyama, Delta unknotting operation and vertex homotopy of graphs in $\mathbf{R}^{3}$, from: "KNOTS '96 (Tokyo)", (S Suzuki, editor), World Sci. Publ., River Edge, NJ (1997) 185-200 MR1664961

[10] H Murakami, Y Nakanishi, On a certain move generating link-homology, Math. Ann. 284 (1989) 75-89 MR995383

[11] R Nikkuni, Delta edge-homotopy invariants of spatial graphs via disk-summing the constituent knots, to appear in Illinois J. Math. arXiv:math.GT/0703319

[12] Y Ohyama, T Tsukamoto, On Habiro's $C_{n}$-moves and Vassiliev invariants of order $n$, J. Knot Theory Ramifications 8 (1999) 15-26 MR1673893

[13] M Okada, Delta-unknotting operation and the second coefficient of the Conway polynomial, J. Math. Soc. Japan 42 (1990) 713-717 MR1069853

[14] N Robertson, P Seymour, R Thomas, Sachs' linkless embedding conjecture, J. Combin. Theory Ser. B 64 (1995) 185-227 MR1339849

[15] H Sachs, On spatial representations of finite graphs, from: "Finite and infinite sets, Vol. I, II (Eger, 1981)", (A Hajnal, L Lovász, V T Sòs, editors), Colloq. Math. Soc. János Bolyai 37, North-Holland, Amsterdam (1984) 649-662 MR818267

[16] M Scharlemann, A Thompson, Detecting unknotted graphs in 3-space, J. Differential Geom. 34 (1991) 539-560 MR1131443

[17] T Soma, H Sugai, A Yasuhara, Disk/band surfaces of spatial graphs, Tokyo J. Math. 20 (1997) 1-11 MR1451853 
[18] K Taniyama, Cobordism, homotopy and homology of graphs in $\mathbf{R}^{3}$, Topology 33 (1994) 509-523 MR1286929

[19] K Taniyama, A Yasuhara, Realization of knots and links in a spatial graph, Topology Appl. 112 (2001) 87-109 MR1815273

[20] Y Q Wu, On planarity of graphs in 3-manifolds, Comment. Math. Helv. 67 (1992) 635-647 MR1185812

[21] H Yamada, Delta distance and Vassiliev invariants of knots, J. Knot Theory Ramifications 9 (2000) 967-974 MR1780599

Institute of Human and Social Sciences, Faculty of Teacher Education, Kanazawa University Kakuma-machi, Kanazawa, Ishikawa, 920-1192, Japan

nick@ed.kanazawa-u.ac.jp

http://www.ed.kanazawa-u.ac.jp/ nick/index-e.html

Received: 30 July 2008 Revised: 29 January 2009 